\title{
Differences Between the International Temperature Scales of 1948 and 1927
}

\author{
By Robert J. Corruccini
}

\begin{abstract}
Tables and a graph are presented showing differences that exist between temperatures expressed on the International Temperature Scale of 1927 and the recently adopted International Temperature Scale of 1948.
\end{abstract}

The experimental difficulties inherent in the measurement of temperature on the thermodynamic scale led to the adoption in 1927 , by the Seventh General Conference on Weights and Measures, of a practical scale that was designated as the International Temperature Scale. ${ }^{1}$ The first revision of this scale came with the adoption of the International Temperature Scale of 1948 [2] ${ }^{2}$ by the Ninth General Conference on Weights and Measures. The revised scale was designed to conform as nearly as practicable to the thermodynamic centigrade (Celsius) scale as then known, while incorporating certain refinements, based on experience, to make the scale more uniform and reproducible than its predecessor.

In the following sections, tables, and a graph are presented showing differences that exist between temperatures expressed on the International Temperature Scales of 1948 and 1927.

Platinum resistance thermometer range (oxygen point to freezing point of antimony). The changes ${ }^{3}$ adopted in this range have the effect of making the scale more definite and reproducible but cause little or no change in the numerical values of temperatures.

Standard thermocouple range (freezing point of antimony to the gold point). The change in the

\footnotetext{
${ }^{1} \mathrm{G}$. K. Burgess, The International Temperature Scale, BS J. Research 1, 635 (1928) RP22.

${ }^{2}$ H. F. Stimson, The International Temperature Scale of 1948, J. Research NBS 42, 209 (1949) RP1962.

3 These changes are: (a) The termination of the scale at the oxygen point $\left(-182.970^{\circ} \mathrm{C}\right)$ instead of at $-190^{\circ} \mathrm{C}$, (b) the division of the scale at the freezing point of antimony (about $630.5^{\circ} \mathrm{C}$ ) instead of at $660^{\circ} \mathrm{C}$, (c) the requirement for higher purity of the platinum of the standard resistance thermometer. In addition, a resolution was adopted that the zero of the thermodynamic centigrade scale should be defined as the temperature 0.0100 degree below that of the triple point of pure water.
}

value for the silver point from $960.5^{\circ}$ to $960.8^{\circ} \mathrm{C}$ changes the numerical values of temperatures measured with the standard thermocouple. ${ }^{4} \mathrm{Be}-$ cause both the 1927 and 1948 scales provide for some variation of thermoelectric properties of standard thermocouples, the differences between the two scales in the thermocouple range cannot be stated explicitly. The differences have been calculated for the median standard thermocouple allowed by the International Temperature Scale of 1948 , namely, $E_{A u}=10,300$ microvolts, $E_{A s}=$ 9,117 microvolts, $E_{S b}=5,530$ microvolts, as follows.

The temperature, $t$, is defined by the formula,

$$
E=a+b t+c t^{2},
$$

where $E$ is the electromotive force of a standard thermocouple of platinum and platinum-rhodium alloy when one junction is at $0^{\circ} \mathrm{C}$ and the other is at the temperature, $t$. The constants, $a, b$, and $c$, are to be calculated from measured values of $E$ at the freezing point of antimony and at the silver and gold points. The fixed points will be referred to by subscripts 1, 2, and 3, respectively, in what follows. For a given set of values of $E$, it is desired to determine the effect upon calculated values of $t$ of changing the value assigned to $t_{2}$. Differentiating eq 1 with respect to $t_{2}$ and setting o $E / \partial t_{2}$ equal to zero leads to the following equation:

$$
\partial t / \partial t_{2}=\frac{-\partial a / \partial t_{2}-t \partial b / \partial t_{2}-t^{2} \partial c / \partial t_{2}}{b+2 c t}
$$

\footnotetext{
${ }^{4}$ In addition there are changes of the type mentioned in footnote 3 that cause little or no change in the numerical values of temperatures. These include requirements for higher purity of the platinum of the standard thermocouple and for smaller permissible limits for the electromotive force of the standard thermocouple at the gold point.
} 
where

$$
\begin{aligned}
a & =\frac{E_{1}\left(t_{2} t_{3}{ }^{2}-t_{3} t_{2}{ }^{2}\right)+E_{2}\left(t_{3} t_{1}{ }^{2}-t_{1} t_{3}{ }^{2}\right)+E_{3}\left(t_{1} t_{2}{ }^{2}-t_{2} t_{1}{ }^{2}\right)}{x} \\
b & =\frac{E_{1}\left(t_{2}{ }^{2}-t_{3}{ }^{2}\right)+E_{2}\left(t_{3}{ }^{2}-t_{1}{ }^{2}\right)+E_{3}\left(t_{1}{ }^{2}-t_{2}{ }^{2}\right)}{x} \\
c & =\frac{E_{1}\left(t_{3}-t_{2}\right)+E_{2}\left(t_{1}-t_{3}\right)+E_{3}\left(t_{2}-t_{1}\right)}{x} \\
x & =t_{2} t_{3}{ }^{2}-t_{3} t_{2}{ }^{2}+t_{3} t_{1}{ }^{2}-t_{1} t_{3}{ }^{2}+t_{1} t_{2}{ }^{2}-t_{2} t_{1}{ }^{2} \\
\frac{\partial a}{\partial t_{2}} & =\frac{E_{1}\left(t_{3}{ }^{2}-2 t_{3} t_{2}\right)-E_{3}\left(t_{1}{ }^{2}-2 t_{1} t_{2}\right)}{x}-\frac{a}{x} \frac{\partial x}{\partial t_{2}} \\
\frac{\partial b}{\partial t_{2}} & =\frac{2 t_{2}\left(E_{1}-E_{3}\right)}{x}-\frac{b}{x} \frac{\partial x}{\partial t_{2}} \\
\frac{\partial c}{\partial t_{2}} & =\frac{E_{3}-E_{1}}{x}-\frac{c}{x} \frac{\partial x}{\partial t_{2}} \\
\frac{\partial x}{\partial t_{2}} & =t_{3}{ }^{2}+2 t_{1} t_{2}-t_{1}{ }^{2}-2 t_{3} t_{2} .
\end{aligned}
$$

Inserting the values

gives

$$
\begin{array}{ll}
t_{1}=630.5 & E_{1}=5,530 \\
t_{2}=960.8 & E_{2}=9,117 \\
t_{3}=1,063 & E_{3}=10,300
\end{array}
$$

$$
\frac{\partial t}{\partial t_{2}}=\frac{-226.466+0.5722281 t-0.0003378967 t^{2}}{8.227+0.003309 t} .
$$

\begin{tabular}{|c|c|}
\hline$\stackrel{t}{{ }^{\circ} \mathrm{C} \text { (Int. 1948) }}$ & ${ }^{\circ} \mathrm{C}$ (Int. 1948) $\operatorname{minus}_{1927)}^{\Delta t}{ }^{\circ} \mathrm{C}$ (Int. \\
\hline 630.5 & 0.00 \\
\hline 650 & +.08 \\
\hline 700 & 24 \\
\hline 750 & .35 \\
\hline 800 & 42 \\
\hline 839.5 & $.43(\max )$ \\
\hline 850 & 43 \\
\hline 900 & 40 \\
\hline 950 & .32 \\
\hline 960.8 & .30 \\
\hline 1000 & .20 \\
\hline 1050 & .05 \\
\hline 1063 & .00 \\
\hline
\end{tabular}

Values of $\Delta \mathrm{t}$ obtained by multiplying values of $\partial t / \partial t_{2}$ by $\Delta t_{\mathrm{Ag}}=0.3 \mathrm{deg}$ are given in table 1 and figure 1 .

Calculation indicates that the maximum variation in $\Delta t$ from the values in table 1 , due to the

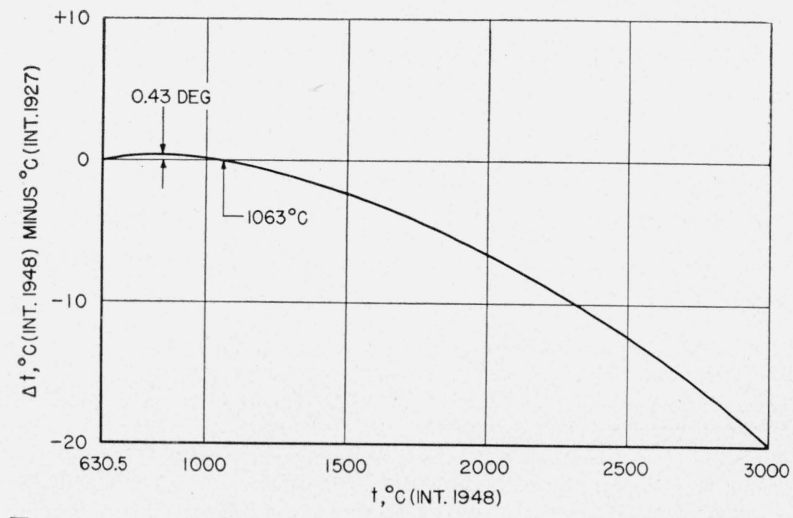

FIGURE 1. Differences between the International [Temperature Scales of 1948 and 1927.
TABLE 1. Differences between the International Temperature Scales of 1948 and 1927 in the standard thermocouple range

variation in standard thermocouples permitted under the International Temperature Scale of 1948 , is $0.002 \mathrm{deg}$. This is sufficient to change certain values of $\Delta t$ by $0.01 \mathrm{deg}$ upon rounding, which is not significant in comparison with the reproducibility of $0.1 \mathrm{deg} \mathrm{C}$ usually ascribed to standard thermocouples. The corresponding temperatures on the two scales between $630.5^{\circ}$ and $1,063^{\circ} \mathrm{C}$ that appear in table 2 were calculated from table 1 .

Radiation law range (above the gold point). The adoption of a different value for the radiation constant $c_{2}$ changes all temperatures above the gold point, while the use of the Planck radiation formula instead of the Wien formula affects the very high temperatures. The Planck formula is consistent with the thermodynamic scale and consequently removes the upper limit that was imposed by Wien's law in the 1927 scale.

Above the gold point the temperature, $t$, in degrees Centigrade (Int. 1948) is defined by the formula,

$$
\frac{J_{t}}{J_{\mathrm{Au}}}=\frac{e^{\frac{c_{2}}{\lambda\left(t_{\mathrm{Au}}+T_{0}\right)}}-1}{e^{\frac{c_{2}}{\lambda\left(t+T_{0}\right)}}-1},
$$

in which $J_{t}$ and $J_{\mathrm{Au}}$ are the radiant energies per unit wavelength interval at wavelength, $\lambda$, emitted per unit time by unit area of a black body at the 
TABLE 2. Corresponding temperatures on the International Temperature Scales of 1948 and 1927

\begin{tabular}{|c|c|c|c|}
\hline \multicolumn{2}{|c|}{ Degrees C (Int.) } & \multicolumn{2}{|c|}{ Corresponding degrees $\mathrm{F}$} \\
\hline 1948 & 1927 & 1948 & 1927 \\
\hline 630.50 & 630.50 & 1166. 9 & 1166. 9 \\
\hline 650 & 649.92 & 1202 & 1201. 9 \\
\hline 700 & 699. 76 & 1292 & 1291. 6 \\
\hline 750 & 749.65 & 1382 & 1381. 4 \\
\hline 800 & 799.58 & 1472 & 1471. 2 \\
\hline 850 & 849.57 & 1562 & 1561. 2 \\
\hline 900 & 899.60 & 1652 & 1651. 3 \\
\hline 950 & 949.68 & 1742 & 1741.4 \\
\hline 960.80 & 960.50 & 1761. 4 & 1760. 9 \\
\hline 1000 & 999.80 & 1832 & 1831.6 \\
\hline 1050 & 1049. 95 & 1922 & 1921. 9 \\
\hline 1063. 00 & 1063. 00 & 1945. 4 & 1945. 4 \\
\hline 1100 & 1100. 2 & 2012 & 2012 \\
\hline 1200 & 1200.6 & 2192 & 2193 \\
\hline 1300 & 1301. 1 & 2372 & 2374 \\
\hline 1400 & 1401. 7 & 2552 & 2555 \\
\hline 1500 & 1502.3 & 2732 & 2736 \\
\hline 1600 & 1603. 0 & 2912 & 2917 \\
\hline 1700 & 1703.8 & 3092 & 3099 \\
\hline 1800 & 1804. 6 & 3272 & 3280 \\
\hline 1900 & 1905.5 & 3452 & 3462 \\
\hline 2000 & 2006. 4 & 3632 & 3644 \\
\hline 2100 & 2107 & 3812 & 3825 \\
\hline 2200 & 2208 & 3992 & 4007 \\
\hline 2300 & 2310 & 4172 & 4189 \\
\hline 2400 & 2411 & 4352 & 4372 \\
\hline 2500 & 2512 & 4532 & 4554 \\
\hline 2600 & 2613 & 4712 & 4736 \\
\hline 2700 & 2715 & 4892 & 4919 \\
\hline 2800 & 2816 & 5072 & 5102 \\
\hline 2900 & 2918 & 5252 & 5285 \\
\hline 3000 & 3020 & 5432 & 5468 \\
\hline 3100 & 3122 & 5612 & 5651 \\
\hline 3200 & 3223 & 5792 & 5834 \\
\hline 3300 & 3325 & 5972 & 6018 \\
\hline 3400 & 3428 & 6152 & 6202 \\
\hline 3500 & 3530 & 6332 & 6386 \\
\hline 3600 & 3632 & 6512 & 6570 \\
\hline 3700 & 3735 & 6692 & 6754 \\
\hline 3800 & 3837 & 6872 & 6939 \\
\hline 3900 & 3940 & 7052 & 7124 \\
\hline 4000 & 4043 & 7232 & 7309 \\
\hline 4100 & 4146 & 7412 & 7495 \\
\hline 4200 & 4249 & 7592 & 7681 \\
\hline
\end{tabular}

temperature, $t$, and at the gold point, $t_{\mathrm{Au}}$, respectively.

$c_{2}=1.438$ centimeter degrees.
$T_{0}=$ temperature of the ice point in degrees Kelvin.

$\lambda=$ wavelength of the visible spectrum.

$e=$ base of Naperian logarithms.

On the International Temperature Scale of 1927 the temperature, $t^{\prime}$, was defined by the formula

$$
\log _{e} \frac{J_{t}}{J_{\mathrm{Au}}}=\frac{c_{2}}{\lambda}\left[\frac{1}{1,336}-\frac{1}{\left(t^{\prime}+273\right)}\right],
$$

where $c_{2}$ is taken as $1.432 \mathrm{~cm}$ degrees and $J_{t}$ and $J_{\text {Au }}$ have the same meanings as in eq 2 . The equation is valid if $\lambda\left(t^{\prime}+273\right)$ is less than $0.3 \mathrm{~cm}$ degree. Equating the logarithm of the right-hand side of eq 2 to the right-hand side of eq 3 , one obtains the following formula relating $t$ in degrees Centigrade (Int. 1948) and $t^{\prime}$ in degrees Centigrade (Int. 1927), in which $t_{\mathrm{Au}}$ is taken as $1,063^{\circ} \mathrm{C}$, $T_{0}$ is taken as 273.16 following the prevailing American usage and $\lambda$ must be expressed in centimeters.

$$
\log _{e} \frac{e^{\frac{1.438}{1336.16 \lambda}}-1}{e^{\lambda(t+273.16)}-1}=\frac{1.432}{\lambda}\left[\frac{1}{1,336}-\frac{1}{\left(t^{\prime}+273\right)}\right] .
$$

Corresponding values of $t$ and $t^{\prime}$ have been calculated from this formula for temperatures above the gold point and for $\lambda=0.65 \times 10^{-4} \mathrm{~cm}$ (which is approximately the effective wavelength of most optical pyrometers) and are given in table 2, together with corresponding temperatures below the gold point obtained as mentioned on p. 134. In figure 1, the difference, degrees Centigrade (Int. 1948) minus degrees Centigrade (Int. 1927), is plotted as a function of degrees Centigrade (Int. 1948). Values below $1,063^{\circ} \mathrm{C}$ are from table 1 .

In the range above the gold point, the differences between the two scales cannot be stated explicitly due to the range of permissible values of $\lambda$. To indicate the possible variation of the differences due to variation of $\lambda$, table 3 has been prepared giving corresponding values of $t$ and $t^{\prime}$ at $\lambda=0.65 \times 10^{-4} \mathrm{~cm}$ and $\lambda=0.4738 \times 10^{-4} \mathrm{~cm}$. The latter wavelength occupies a position at the blue end of the visibility function of the human eye, which corresponds to the position of $\lambda=$ $0.65 \times 10^{-4} \mathrm{~cm}$ at the red end. It will be noted that the variation in difference due to variation of $\lambda$ is in all cases much less than the accuracy of measurement of temperature with existing instruments. 
Tables 2 and 3 have not been carried above the maximum temperatures to which the International Temperature Scale of 1927 extends, namely, $4,342^{\circ} \mathrm{C}$ for $\lambda=0.65 \times 10^{-4} \mathrm{~cm}$ and $6,059^{\circ} \mathrm{C}$ for $\lambda=0.4738 \times 10^{-4} \mathrm{~cm}$.

TABLE 3. Corresponding temperatures on the Internationa? Temperature Scales of 1948 and 1927 at two wave lengths

\begin{tabular}{|c|c|c|}
\hline \multirow{2}{*}{$\begin{array}{c}t \\
{ }^{\circ} \mathrm{C} \text { (Int. 1948) }\end{array}$} & \multicolumn{2}{|c|}{${ }^{\circ} \mathrm{C}$ (Int. 1927) } \\
\cline { 2 - 3 } & ${ }^{t^{\prime}}$ \\
\hline & $\left.\lambda=0.65 \times 10^{-4} \mathrm{~cm}\right)$ & $\left(\lambda=0.4738 \times 10^{-4} \mathrm{~cm}\right)$ \\
\hline 1063 & 1063 & 1063 \\
1500 & 1502.31 & 1502.31 \\
2000 & 2006.41 & 2006.39 \\
2500 & 2512.14 & 2512.02 \\
3000 & 3019.76 & 3019.22 \\
3500 & 3529.79 & 3528.07 \\
4000 & 4042.99 & 4038.72 \\
5000 & $-\ldots . .-$ & 5066.51 \\
& & \\
\hline
\end{tabular}

In determining temperatures with an optical pyrometer, the following approximate formula (based upon Wien's law),

$$
\log _{e} \frac{J_{t}}{J_{A u}}=\frac{1.438}{\lambda}\left[\frac{1}{1,336}-\frac{1}{\left(t_{w}+273\right)}\right]
$$

will usually yield values that are not significantly different from those yielded by eq 2 . Corresponding values of $t$, degrees Centigrade (Int. 1948) and $t_{w}$ are given in table 4 .

TABLE 4.-Corresponding temperatures on the International Temperature Scale of 1948 and from eq 4

\begin{tabular}{|c|c|}
\hline \multirow{2}{*}{$\begin{array}{c}t \\
{ }^{t} \text { (Int. 1948) }\end{array}$} & ${ }^{{ }^{t} \mathrm{C}}$ \\
\hline & \\
\hline 1063 & 1063.0 \\
1500 & 1499.9 \\
2000 & 1999.7 \\
2500 & 2499.6 \\
3000 & 2999.8 \\
3500 & 3500.7 \\
4000 & 4003.2 \\
4500 & 4508.2 \\
5000 & 5016.8 \\
\hline
\end{tabular}

Acknowledgment is due members of the Computation Laboratory of this Bureau and John I. Lauritzen, who have carried out most of the calculations involved in this work.

Washington, March 2, 1949. 